\title{
X-ray Emission from O Stars
}

\author{
David H. Cohen \\ Swarthmore College, Department of Physics and Astronomy, 500 College Ave., Swarthmore, \\ Pennsylvania 19081, USA
}

\begin{abstract}
Young O stars are strong, hard, and variable X-ray sources; properties that strongly affect their circumstellar and galactic environments. After $\approx 1 \mathrm{Myr}$, these stars settle down to become steady sources of soft X-rays. I will use high-resolution X-ray spectroscopy and MHD modeling to show that young $\mathrm{O}$ stars like $\theta^{1}$ Ori $\mathrm{C}$ are well explained by the magnetically channeled wind shock scenario. After their magnetic fields dissipate, older O stars produce Xrays via shock heating in their unstable stellar winds. Here too I will use X-ray spectroscopy and numerical modeling to confirm this scenario. In addition to elucidating the nature and cause of the $\mathrm{O}$ star X-ray emission, modeling of the high-resolution X-ray spectra of $\mathrm{O}$ supergiants provides strong evidence that mass-loss rates of these $\mathrm{O}$ stars have been overestimated.
\end{abstract}

Keywords. hydrodynamics - instabilities - line: profiles - magnetic fields - MHD - shock waves - stars: early type - stars: mass loss - stars: winds, outflows - X-rays: stars

O stars dominate the X-ray emission from young clusters, with X-ray luminosities up to $L_{\mathrm{x}}=10^{34} \mathrm{ergs} \mathrm{s}^{-1}$ and emission that is hard (typically several $\mathrm{keV}$ ) and often variable. This strong X-ray emission has an effect on these $\mathrm{O}$ stars' environments, including nearby sites of star formation and protoplanetary disks surrounding nearby low-mass premain-sequence stars. The X-ray emission is also interesting in its own right, as it traces important high-energy processes in the extended atmospheres of $\mathrm{O}$ stars. In this paper, I will show how the spectral properties of the X-rays from the prototypical young, magnetized $\mathrm{O}$ star, $\theta^{1}$ Ori $\mathrm{C}$ are in line with the predictions of the Magnetically Channeled Wind Shock (MCWS) model, but how this process seems to dissipate as $\mathrm{O}$ stars age, with weaker line-driven instability wind shocks explaining the X-ray emission in older O stars. I also will show how the X-ray emission can be used as a probe of the conditions in the bulk stellar winds of these objects. Specifically, the resolved X-ray line profiles in normal $\mathrm{O}$ supergiants provide an independent line of evidence for reduced mass-loss rates.

Now, it is certainly the case that many young O stars do not show the X-ray signatures of the MCWS mechanism. And indeed, only a handful of $\mathrm{O}$ and early B stars have had direct detections of magnetic fields. Of course, highly structured, non-dipole fields will be very difficult to detect on hot stars, even if their local strength is quite high. But one should keep in mind that wind-wind interactions in close binaries can also produce the hard, strong, and variable $\mathrm{X}$-rays seen in many young $\mathrm{O}$ stars.

Because $\theta^{1}$ Ori $\mathrm{C}$ has a well established, predominantly dipole field, and because many of its properties are explained by this field, I treat it here as a potential prototype. I also note that the incidence of hard, strong X-ray emission from $\mathrm{O}$ stars diminishes rapidly as one looks from young $(<1 \mathrm{Myr})$ clusters to older $(2$ to $5 \mathrm{Myr})$ clusters. This fact can be explained if the fossil fields in young $\mathrm{O}$ stars dissipate as the stars age. If wind-wind binaries account for many of these sources, then only the very earliest $\mathrm{O}$ stars with very short lifetimes are involved.

We begin the comparison of young and old $\mathrm{O}$ stars and their X-rays by showing in Fig. 1 the Chandra MEG spectra of two representative stars: $\theta^{1}$ Ori C (O4-7 V), with 
an age of $\approx 1 \mathrm{Myr}$, and $\zeta$ Pup (O4 If), with an age of several Myr, and already evolved well off the main sequence. The $\zeta$ Pup X-ray spectrum is typical of those measured for most O stars.

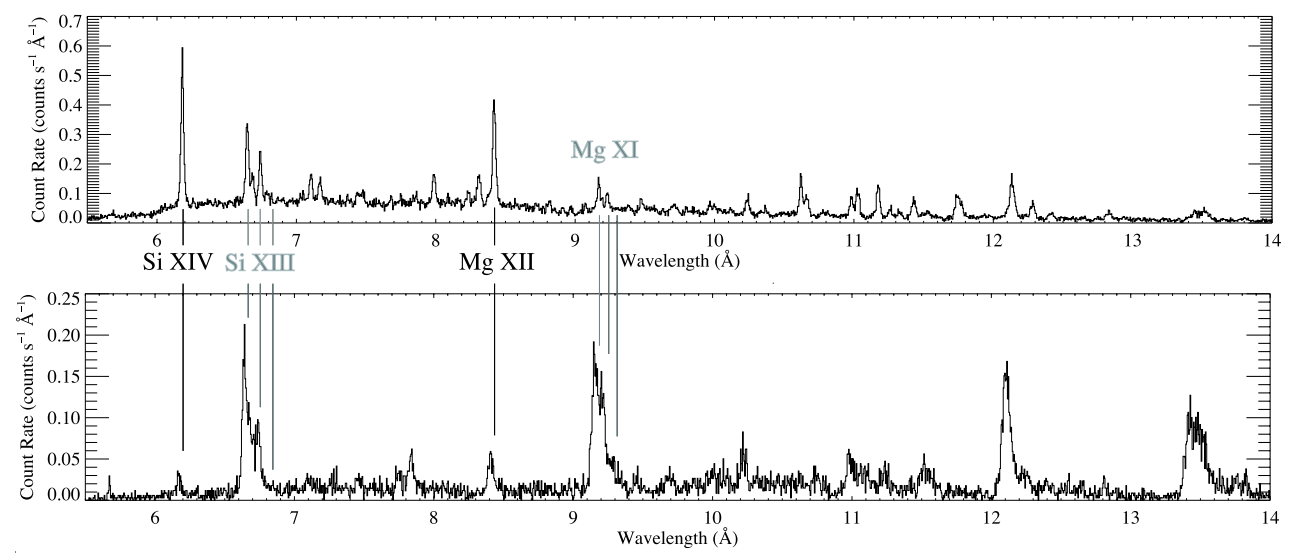

Figure 1. Chandra MEG spectra of $\theta^{1}$ Ori C (top) and $\zeta$ Pup (bottom). The hydrogen-like Lyman alpha lines of $\mathrm{Si}$ and $\mathrm{Mg}$ are indicated in black, while the helium-like resonance-intercombination-forbidden complexes of the same elements are indicated in gray.

Two obvious differences between these spectra are the hardness of the X-ray emission from $\theta^{1}$ Ori $\mathrm{C}$ and the small line widths in that star's spectrum. The hardness implies a much higher plasma temperature in the young $\mathrm{O}$ star, and this is best seen in the data when one compares resonance lines of hydrogen-like and helium-like ionization states of abundant elements. In Fig. 1 I have labeled these lines for silicon and magnesium. In $\theta^{1}$ Ori $\mathrm{C}$ the hydrogen-like lines are much stronger, whereas in $\zeta$ Pup the helium-like lines are stronger. This reflects a significantly different ionization balance in these two stars which is a direct effect of their different plasma temperatures. The plasma temperature distribution, based on the analysis of high-resolution Chandra spectra of several O stars, has been determined by Wojdowski \& Schulz (2005). These authors assume a continuous Differential Emission Measure (DEM), which can be thought of as a density-squared weighting of the plasma temperature distribution. Their results are shown in Fig. 2, where it can easily be seen that the DEM for $\theta^{1}$ Ori $\mathrm{C}$ is the only one (of seven $\mathrm{O}$ stars) with a positive slope. Its peak is near $T=30$ million $\mathrm{K}$, whereas the DEMs of the more evolved O stars (including $\zeta$ Pup) all peak near one or two million $\mathrm{K}$.

We also show in Fig. 2 the DEM from a snapshot of an MHD simulation of the magnetically confined wind of $\theta^{1}$ Ori $\mathrm{C}$. The agreement is quite good, both in terms of the overall emission measure and the shape of the DEM. The simulation shows a peak at 15 to 20 million $\mathrm{K}$, modestly lower than that seen in the data, but the main property - a rising DEM up to and beyond 10 million $\mathrm{K}$ - matches the data well. These MHD simulations confirm the predictions of Babel \& Montmerle (1997) that strong shock fronts near the magnetic equator where the wind from the northern and southern hemispheres meets can heat a significant amount of wind plasma to the observed high temperatures. Snapshots of temperature and emission measure from an MHD simulation are shown in Fig. 3. It can be seen that the bulk of the shock-heated plasma is in the magnetically confined region near $r \approx 2 \mathrm{R}_{*}$. Due to this confinement, the speed and line-of-sight velocity of this material is low, and thus the emission lines from the thermal $\mathrm{X}$-ray emission are relatively narrow. 

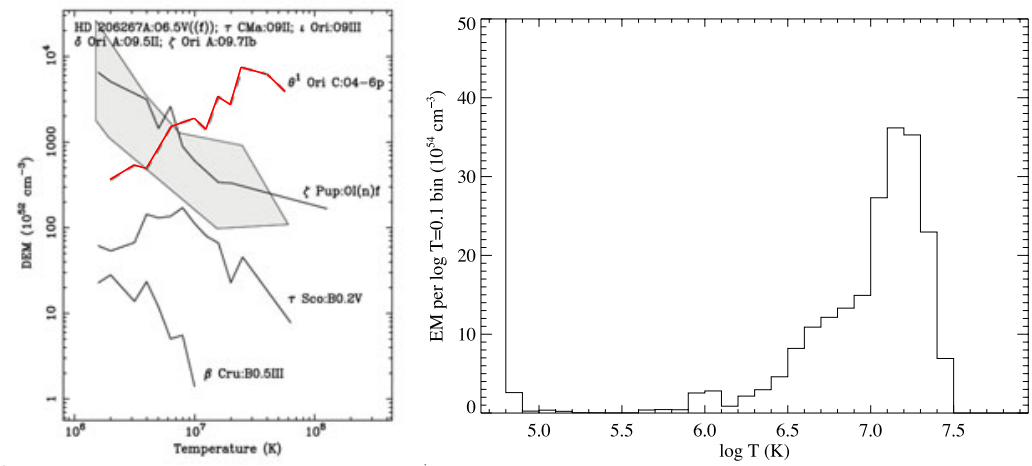

Figure 2. Differential emission measures of seven O stars and two B stars, derived from thermal spectral model fits to Chandra spectra (left; taken from Wojdowski \& Schulz, 2005). $\theta^{1}$ Ori C is the only star whose DEM has a positive slope. The panel on the right shows a DEM predicted by the MHD simulations of $\theta^{1}$ Ori C (taken from Gagné et al., 2005).
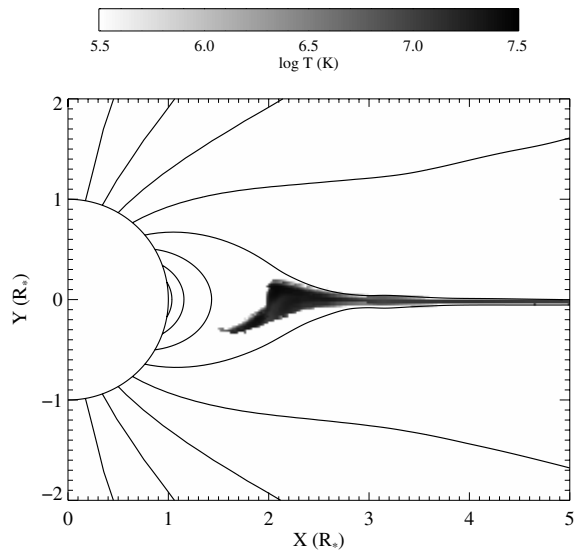
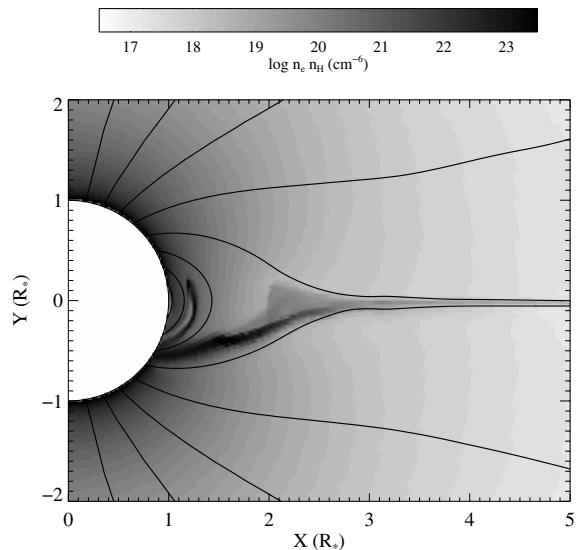

Figure 3. Contour plots of temperature (left) and emission measure per unit volume (right) from a 2-D MHD simulation of the magnetically confined wind of $\theta^{1}$ Ori $\mathrm{C}$ (taken from Gagné et al., 2005). Magnetic field lines are displayed as contours. The wind flow up each closed field line encounters a strong shock due to the ram pressure of the wind flow from the opposite hemisphere, which heats the plasma according to $T_{\text {shock }} \approx 10^{7}\left(v_{\text {shock }} / 1000 \mathrm{~km} \mathrm{~s}^{-1}\right)^{2} \mathrm{~K}$. The head-on nature of the wind shocks leads to high shock velocities and temperatures. In these MHD models, the field configuration is self-consistently solved for along with the wind dynamics. Note that another difference between the MHD simulations of the MCWS model and the initial analysis of Babel \& Montmerle (1997) is the dynamical infall of material from the magnetic equator. Evidence of this can be seen in the snake-like structure visible in the emission measure panel, just above the star's surface, slightly below the equator.

In contrast, the X-ray emission lines of mature $\mathrm{O}$ stars, like $\zeta$ Pup, are quite broad, as can be seen in Fig. 4, where I show the neon Ly $\alpha$ lines for the two stars. The Xray spectrum of $\zeta$ Pup is also soft, as I have already shown. The X-ray emission from these older, presumably non-magnetized, $\mathrm{O}$ stars is thought to arise in much milder wind shocks, embedded in the outflowing, highly supersonic line-driven winds. The LineDriven Instability (LDI) is generally thought to produce these shocks, although models have difficulty reproducing the overall level of X-ray emission unless the instability is seeded, perhaps by sound waves injected at the base of the wind (Feldmeier et al., 1997). 
The softness of the X-ray spectra, along with the large line widths from the high velocity of the shock-heated wind, is well explained by this LDI wind-shock scenario, as I show in Fig. 5. This figure shows a snapshot from a 1-D radiation hydrodynamics simulation of the wind of an $\mathrm{O}$ supergiant like $\zeta$ Pup, accounting for non-local line radiation transport. The instability grows rapidly beyond about half a stellar radius (in height; $r=1.5 \mathrm{R}_{*}$ ). Shock fronts can be seen in this snapshot, but they typically have velocity jumps of only a few hundred $\mathrm{km} \mathrm{s}^{-1}$, leading to heating of only a few million $\mathrm{K}$. The soft spectrum seen in Fig. 1 and the DEMs weighted to low plasma temperatures, shown in the left-hand panel of Fig. 2, are in line with the results of this simulation.
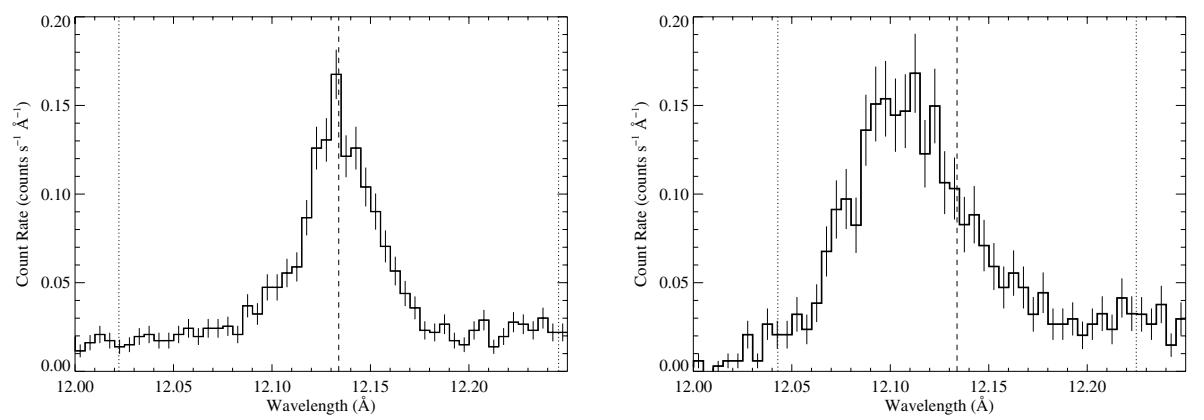

Figure 4. Ne X Lyman alpha lines in the Chandra MEG spectra of $\theta^{1}$ Ori C (left) and $\zeta$ Pup (right). The vertical dashed line represents the laboratory rest wavelength of this transition, and the vertical dotted lines represent the blue and red shifts associated with the UV wind terminal velocity in each star. Note that the profile in the $\zeta$ Pup spectrum is shifted and skewed as well as being broadened. Error bars are from Poisson photon-counting statistics.

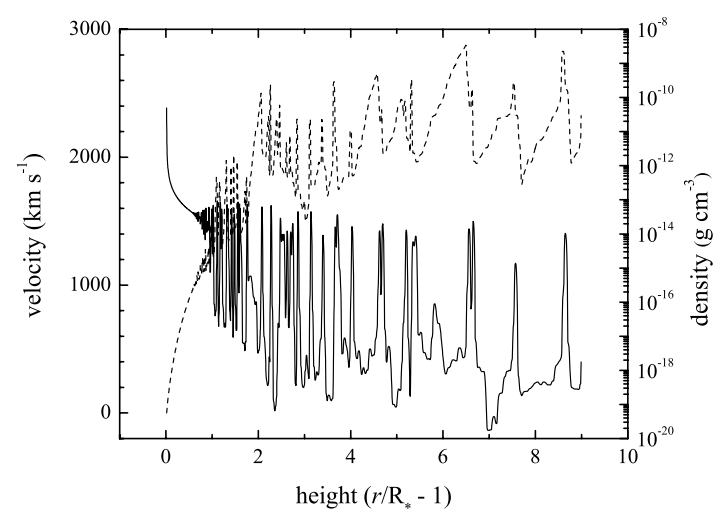

Figure 5. A snapshot showing the velocity (dashed line; left-hand axis) and density (solid line; right-hand axis) as a function of height above the photosphere in a radiation hydrodynamics simulation of the wind of $\zeta$ Pup. Shock-heated plasma cools rapidly; the bulk of the wind in any given snapshot is cold.

The predictions of this model can be further tested by examining the X-ray emission line profiles, which have a characteristic asymmetric, skewed shape, as can be seen in the right-hand panel of Fig. 4. This shape, with a deficit of red-shifted photons, arises in a spherically expanding wind with hot, line-emitting material intermixed with warm, 
continuum-absorbing material, as motivated by the global structure seen in Fig. 5. If the wind is optically thick (in the continuum; $\kappa \approx$ constant across a line), then there is significantly more attenuation of emission from the back of the wind, which is the red shifted portion. And there is comparatively more emission from the front, blue shifted, less attenuated, side.

I fit an empirical model (Owocki \& Cohen, 2001) to emission lines in the spectrum of $\zeta$ Pup and get good fits by adjusting only three parameters: the normalization, the inner radius below which there is assumed to be no emission $\left(R_{0}\right)$, and the wind optical depth (parameterized by the quantity $\tau_{*} \equiv \dot{M} \kappa / 4 \pi R_{*} v_{\infty}$ ). The fit to the Fe XVII line at 15.014 $\AA$ is typical and is shown in Fig. 6 as the dashed histogram. The fit is formally good, and the best-fit values with joint $68 \%$ parameter confidence limits are $R_{0}=1.53_{-0.15}^{+0.12} \mathrm{R}_{*}$ and $\tau_{*}=2.0 \pm 0.4$.

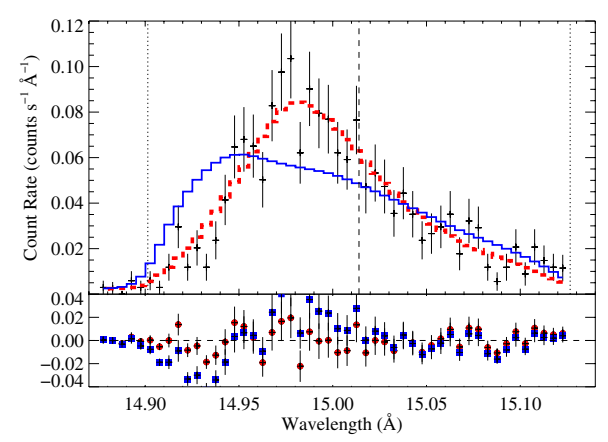

Figure 6. Best-fit wind profile model, for a non-porous wind, (dashed histogram) fit to the Fe XVII line in the Chandra spectrum of $\zeta$ Pup. The solid histogram is the best-fit non-porous model for which the optical depth parameter is fixed at the value implied by the literature mass-loss rate $\left(\tau_{*}=8\right)$. Fit residuals for the two models are shown in the lower panel, with the circles corresponding to the low optical depth model represented by the dashed histogram, and the squares corresponding to the higher optical depth model.

The $R_{\mathrm{o}}$ derived from the profile is consistent with the onset of the self-excited instability seen in the hydrodynamics simulation shown in Fig. 5. The optical depth consistent with the data is actually quite small, which can be seen qualitatively in the relatively modest asymmetry. The mass-loss rate of $6 \times 10^{-6} \mathrm{M}_{\odot} \mathrm{yr}^{-1}$ derived from $\mathrm{H} \alpha$ emission (Puls et al., 1996) implies $\tau_{*}=8$. The best-fit model with that value fixed is shown in Fig. 6 as the solid histogram. The fit is formally very poor. Thus it would appear that the X-ray line profiles provide independent evidence that mass-loss rates of $\mathrm{O}$ stars must be revised downward by a factor of several; a factor of 4 for this star, according to this particular line $\left(\tau_{*}=2.0 \pm 0.4\right.$ vs. $\left.\tau_{*}=8\right)$.

It has been suggested that porosity associated with large-scale clumping - rather than reduced mass-loss rates - can account for the surprisingly small degree of asymmetry in the observed X-ray emission line profiles in O stars (Oskinova et al., 2006). By fitting the line profile model, modified for the effects of porosity produced by spherical clumps (Owocki \& Cohen, 2006), I can quantify the trade-off between atomic opacity and porosity (see also Cohen et al., 2008). In Fig. 7 I show the best-fit porous model with $\tau_{*}=8$, the value implied by the literature mass-loss rate, and with the porosity length, $h \equiv \ell / f$, free to vary. Here $\ell$ is the clump size and $f$ is the volume filling factor of the clumps. The porosity length, $h$, completely describes the effects of porosity on line profiles and in the limit of small clumps it is equivalent to the interclump spacing. The best-fit porous model 
D. H. Cohen

with the literature mass-loss rate is nearly indistinguishable from the best-fit non-porous model, although the fit quality is formally not as good. More importantly, it requires a terminal porosity length (the value of $h$ in the outer portion of the wind) of at least $h_{\infty}=2.5 \mathrm{R}_{*}(68 \%$ confidence $)$, as shown in the right-hand panel of Fig. 7 . Even ignoring the worse quality of the porous model fits, the very high values of the porosity length required to fit the data are vastly larger than the porosity lengths seen in state-of-the-art 2-D radiation hydrodynamics simulations, which show LDI-induced structure down to the grid scale (Dessart \& Owocki, 2005).Therefore, I conclude that there is no compelling evidence that porosity explains the modestly asymmetric X-ray line profiles in $\zeta$ Pup. Rather, these profiles provide independent evidence for a reduced mass-loss rate.
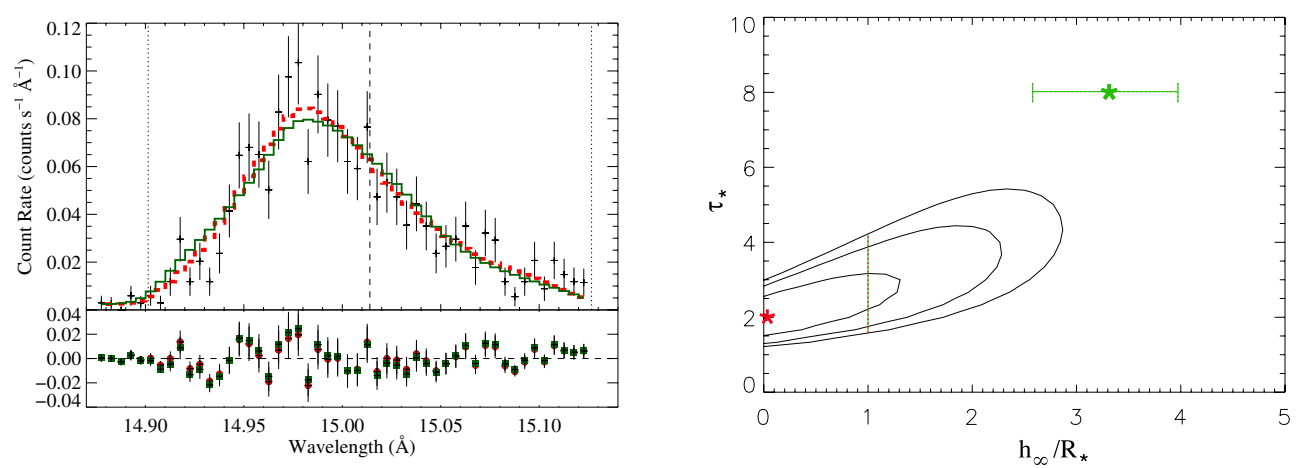

Figure 7. Best-fit wind profile model, for a non-porous wind (dashed histogram), fit to the Fe XVII line in the Chandra spectrum of $\zeta$ Pup (left). The solid histogram is the best-fit porous model for which the optical depth parameter is fixed at the value implied by the literature mass-loss rate $\left(\tau_{*}=8\right)$. We also show confidence limits $(68,90,95 \%)$ in $h_{\infty}$ vs. $\tau_{*}$ parameter space (right). The global best-fit model is indicated by the star at $h_{\infty}=0, \tau_{*}=2$. The star at $h_{\infty}=3.3, \tau_{*}=8$ represents the best-fit model with the wind optical depth fixed at the value implied by the literature mass-loss rate. The horizontal bar centered on it represents the $68 \%$ confidence limit on the value of $h_{\infty}$, given $\tau_{*}=8\left(2.5<h_{\infty} / \mathrm{R}_{*}<4.0\right)$. The vertical line at $h_{*}=1$ emphasizes that even that large porosity length cannot bring the optical depth close to the value associated with the literature mass-loss rate.

\section{Acknowledgements}

I acknowledge support from NASA/CXC grant AR7-8002X and from the Hungerford Faculty Support Endowment of Swarthmore College's provost's office.

\section{References}

Babel, J. \& Montmerle, T. 1997, ApJ, 485, L29

Cohen, D. H., Leutenegger, M., \& Townsend, R. H. D. 2008, in: W.-R. Hamann, A. Feldmeier, L. M. Oskinova (eds.), Clumping in Hot Star Winds (Potsdam: Universitäts-Verlag)

Dessart, L. \& Owocki, S. P. 2005, ApJ, 437, 657

Feldmeier, A., Puls, J., \& Pauldrach 1997, A\& A, 322, 878

Gagné, M., Oksala, M., Cohen, D. H., et al. 2005, ApJ, 628, 986

Oskinova, L. M., Feldmeier, A., \& Hamann, W.-R. 2006, MNRAS, 372, 313

Owocki, S. P. \& Cohen, D. H. 2001, ApJ, 559, 1108

Owocki, S. P. \& Cohen, D. H. 2006, ApJ, 648, 565

Puls J., Kudritzki, R.-P., Herrero, A., et al. 1996, A\&̈A, 305, 171

Wojdowski, P. \& Schulz, N. S. 2005, ApJ, 627, 953 


\section{Discussion}

KUDRITZKI: It is very important to repeat the observing experiment that you have done for $\theta^{1}$ Ori $\mathrm{C}$ for young $\mathrm{O}$ stars, which are more massive and luminous than $\theta^{1}$ Ori $\mathrm{C}$. I speculate that for those objects, the magnetic focusing of the stellar winds will be less effective, because the winds are stronger and the ratio of magnetic to mechanical wind energy is lower. I think it is really crucial to do such observations.

Cohen: I agree. The degree of channeling and confinement, however, goes as $\mathrm{B}^{2}$ but only as $1 / \dot{M}$, so the extent of channeling and confinement in any given star is more likely to be dominated by trends in magnetic field strength, which we don't understand, than by trends in mass-loss rate associated with stellar mass and luminosity. I think the key measurements to make in order to test the idea that the MCWS mechanism on $\theta^{1}$ Ori $\mathrm{C}$ is a paradigm for $\mathrm{X}$-ray emission in young $\mathrm{O}$ stars would be Zeeman measurements of fields on the massive cluster stars that are known to be strong, hard X-ray sources. High-resolution X-ray spectroscopy would also be very useful, obviously, if it's feasible. As I discussed in my talk in the special session on magnetic massive stars on Sunday, line widths and helium-like forbidden-to-intercombination line strength ratios provide information beyond what's provided by CCD-based (e.g. Chandra ACIS or XMM EPIC) X-ray data.

ZINNECKER: My question refers to $\theta^{1}$ Ori $\mathrm{C}$ and the origin of the obliquity between its rotational axis and magnetic field axis. From star formation theory it would seem an aligned magnetic rotator would be expected. Any suggestions to explain the misalignment?

CoHen: I don't have any special expertise in star formation theory, but if models predict aligned rotation and magnetic axes, then there must be some important physics missing from them. Many of the magnetic massive stars have highly misaligned axes: $\tau$ Sco, $\beta$ Cep, and $\sigma$ Ori E, for example, are all close to $\beta=90$ degrees.

Skinner: A new paper by M. Güdel et al. (2008 Sci 319, 309) reports the first detections of hot (1 to $2 \mathrm{MK}$ ) diffuse X-ray emission in the extended Orion Nebula. This article argues that massive Trapezium O stars (and their shocked winds) are ultimately responsible for the diffuse X-ray emission detected by XMM-Newton.

CoHen: I think that the problem of heating the diffuse, X-ray emitting plasma in massive star clusters is a hard one. The shocked wind (such as $\zeta$ Pup's) will adiabatically cool over distances much less than $1 \mathrm{pc}$. And the morphology of the X-rays isn't what you'd expect from the wind slamming into dense interstellar gas at the boundaries of these cavities.

WALBORN: There are currently only two O stars with observed magnetic fields, $\theta^{1}$ Ori $\mathrm{C}$ and HD 191612. The latter can be understood as a spun-down version of the former, with a rotational period of $538 \mathrm{~d}$ (vs. $15 \mathrm{~d}$ for $\theta^{1}$ Ori C), a soft X-ray spectrum, and an age of 3 to $4 \mathrm{Myr}$. Nevertheless, it is a very unusual object, with a very peculiar spectrum and extreme, periodic spectral variations. So these two stars are inconsistent with your hypothesis that $\theta^{1}$ Ori $\mathrm{C}$ is a typical very young $\mathrm{O}$ star, and that magnetic fields have disappeared at $\sim 5$ Myr. I think that both of these objects are unusual areas of large fossil fields. An alternate interpretation of your cluster X-ray differences might be different frequencies of wind-wind collision binaries. 
CoHen: While I agree than wind-wind collision binaries may make a significant contribution to the observed population of hard, strong X-ray sources in young clusters, I think we need more information about the highly unusual O star, HD 191612, not to mention more positive detections or strong upper limits to magnetic field strengths in other $\mathrm{O}$ stars, both young and old. The X-ray spectrum of HD 191612 looks like that of a typical, older $\mathrm{O}$ star, with very broad lines and an SED that is quite soft. Perhaps the fields of young $\mathrm{O}$ stars like $\theta^{1}$ Ori $\mathrm{C}$ become more spatially structured as they evolve. It's possible that HD 191612 has a field that looks more like that of tau Sco and is not dominated by a large scale dipole. If that's the case, then there may not be any large-scale confinement and channeling of the stellar wind, and no substantial MCWS mechanism. The X-ray emission may instead arise in open field regions, in a loose analogy to the solar wind and coronal holes.

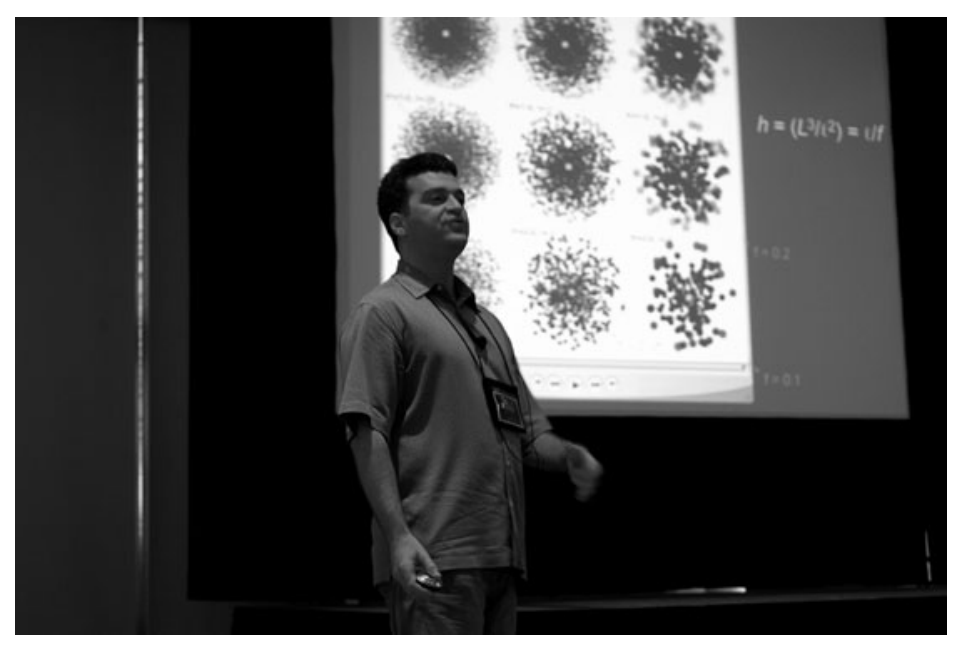

David Cohen.

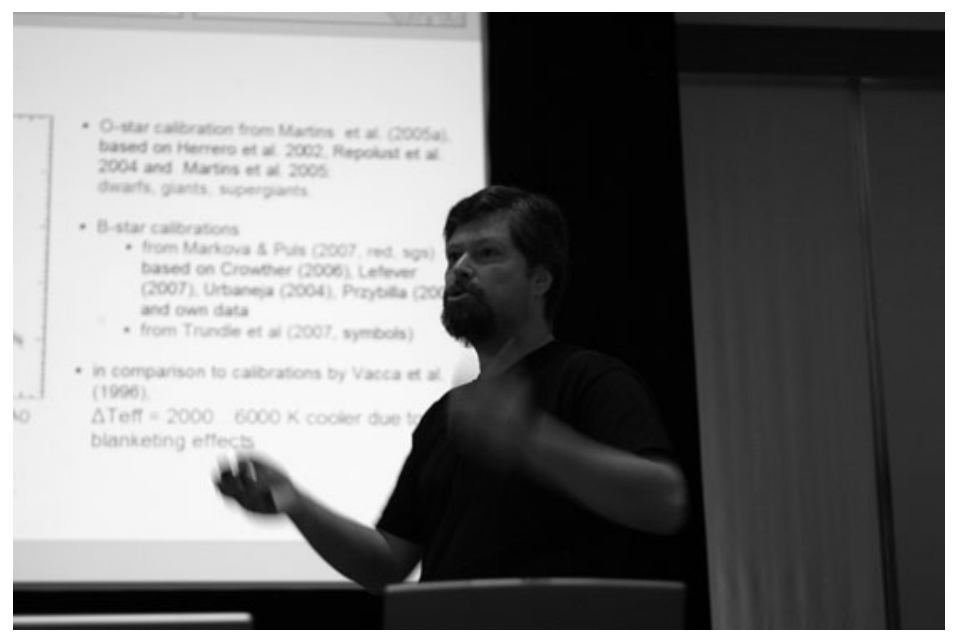

Joachim Puls. 